\title{
Dimetilarginina asimétrica plasmática en mujeres obesas y no obesas con síndrome de ovarios poliquísticos.
}

\author{
Jorly Mejia Montilla ${ }^{1}$, Melchor Álvarez-Mon ${ }^{2}$, Eduardo ReynaVillasmil 1*, Duly TorresCepeda ${ }^{1}$,

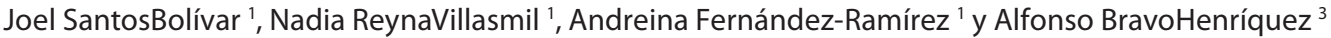

Resumen: Objetivo: determinar las concentraciones plasmáticas de factor de dimetilarginina asimétrica (ADMA) en mujeres obesas y no obesas con diagnóstico de síndrome de ovarios poliquísticos (SOPQ). Métodos: se realizó un estudio de casos y controles en mujeres con diagnóstico de SOPQ y controles sanas de edades similares, con menstruaciones regulares y ovarios normales por ecografía y fueron divididas en cuatro grupos (grupo A: SOPQ obesas; grupo B: SOPQ no obesas; grupo C: controles obesas y grupo D controles no obesas) de acuerdo con el índice de masa corporal (obesas $>30 \mathrm{Kg} / \mathrm{m}^{2}$ y no obesas $<25 \mathrm{~kg} / \mathrm{m}^{2}$ ). Se midieron las concentraciones de hormonas sexuales, globulina fijadora de hormonas sexuales, glucosa sérica, insulina y ADMA. Resultados: las mujeres con SOPQ obesas y no obesas presentaron concentraciones más elevadas de hormonas sexuales e insulina comparadas con el grupo control de obesas y no obesas $(p<0,0001)$. Se observó que las mujeres con SOPQ presentaron concentraciones significativamente más altas de ADMA (grupo A: 0,56 +/- 0,05 picomol/L y grupo B: 0,51 +/- 0,03 picomol/L) comparado con los controles (grupo C: 0,47 +/- 0,02 picomol/L y grupo D $0,45+/-0,04 \mathrm{picomol} / \mathrm{L} ; \mathrm{p}<0,0001)$. Se observó que las concentraciones de ADMA presentaban correlación positiva y significativa con los valores de glicemia e insulina en ayunas en las mujeres con SOPQ ( $p<0,0001)$. Conclusión: Existen diferencias significativas en las concentraciones plasmáticas del ADMA entre las mujeres con SOPQ obesas y no obesas respecto a los controles normales.

Palabras clave: síndrome de ovarios poliquísticos; dimetilarginina asimétrica; obesidad.

Abstract: Objective: To determine plasma concentrations of asymmetric dimethylarginine (ADMA) in obese and non-obese women with polycystic ovary syndrome (PCOS). Methods: A Case control study was done of women with diagnosis of PCOS and age-matched healthy controls, regular menstruations and normal ultrasound ovaries were selected Participants were divided in four groups (group A: PCOS and obese; group B: PCOS and non-obese; group C: obese controls and group D non-obese controls) according to body mass index (obese $>30 \mathrm{Kg} / \mathrm{m}^{2}$ y non-obese $<25 \mathrm{~kg} / \mathrm{m}^{2}$ ). Concentrations of sexual hormones, sex hormone-binding globulin, serum glucose, insulin and ADMA. Results: Obese and non-obese women with PCOS had higher luteinizing hormone, follicle stimulating hormone, androstenodione, testosterone, and insulin levels as compared to women in the obese and non-obese control group, respectively ( $p<$ 0.0001). Women with PCOS had significantly higher of of ADMA (group A 0,56 +/- 0.05 picomol/L and group B: $0.51+/-0.03$ picomol/L) as compared with controls (group C: $0.47+/-0.02 \mathrm{picomol} / \mathrm{L}$ and group D $0.45+/-0.04 \mathrm{picomol} / \mathrm{L} ; \mathrm{p}<0.0001$ ). We observed that ADMA concentrations presented a positive and significant correlation with fasting glycaemia and insulin in PCOS women ( $p<0.0001)$. Conclusion: there are significant differences in plasma ADMA concentrations between obese and non-obese women as compared with polycystic ovary syndrome and normal controls.

Keywords: Polycystic ovary syndrome; Asymmetric dimethylarginine; Obesity.

Fecha de envío: 06 de Junio de 2016 - Fecha de aceptación: 30 de Agosto de 2016

\section{Introducción}

El síndrome de ovarios poliquísticos (SOPQ) afecta cerca del 10\% de las mujeres en edad reproductiva y se presenta con manifestaciones reproductivas que incluyen anovulación, infertilidad e hiperandrogenismo. Se conoce que la resistencia a la insulina es un componente fisiopatológico clave, que contribuye al hiperandrogenismo y a los hallazgos metabólicos (Shah \& Rasool, 2016).

(1) Universidad del Zulia. Venezuela, Servicio de Ginecología, Hospital Central "Dr. Urquinaona".

(2) Departamento de Medicina, Universidad de Alcalá, Madrid, España.

(3) Laboratorio de Investigación y Desarrollo en Nutrición, La Universidad del Zulia, Maracaibo, Estado Zulia, Venezuela.

*Autor de Correspondencia: sippenbauch@gmail.com 
El sobrepeso y la obesidad afecta del 50 al $80 \%$ de las mujeres con SOPQ y exacerba la resistencia a la insulina y las características reproductivas y metabólicas (Zhang et al., 2013).

El SOPQ también está asociado con incremento en los factores de riesgo cardiovasculares incluyendo síndrome metabólico, alteración de la tolerancia glucosada, diabetes mellitus no insulinodependiente, disfunción endotelial y aterosclerosis subclínica (Cobin, 2013). La hiperadrogenemia y la resistencia a la insulina están asociados con incremento de la morbilidad metabólica y cardiovascular en mujeres con SOPQ (Vipin et al., 2013). Todas estas alteraciones están asociadas con la disfunción endotelial, lo cual lleva al desarrollo de aterosclerosis subclínica e inflamación vascular crónica de bajo grado (Arikan et al., 2009).

La dimetilarginina asimétrica (ADMA) es un producto del metabolismo proteínico que se forma en todas las células del cuerpo $y$, al bloquear la producción de óxido nítrico (NO), induce la disfunción endotelial, lo que favorece el proceso aterógeno La dimetil-L-arginina simétrica es producida en cantidades equivalentes pero no afecta la producción de ON. El aumento de las concentraciones está asociado con disfunción endotelial (Willeit et al., 2015). En individuos sanos, la relación L-arginina / ADMA se correlaciona con la disfunción endotelial (Lind et al., 2013). Las concentraciones plasmáticas de ADMA se incrementan en diferentes condiciones patológicas como hipercolesterolemia, hipertrigliceridemia e hiperhomocisteinemia (Atzeni et al., 2011; Dimitroulas et al., 2012). Las concentraciones plasmáticas elevadas están asociadas con eventos cardiovasculares (Ari et al., 2010). Además, existe una relación cercana con la resistencia a la insulina.

El objetivo de la investigación fue determinar las concentraciones plasmáticas de dimetilarginina asimétrica entre mujeres obesas y no obesas con diagnóstico de síndrome de ovarios poliquísticos y correlacionar las concentraciones del ADMA con los parámetros de laboratorio bioquimicos y hormonales.

\section{Métodos}

Entre septiembre 2009 y enero 2016, se incluyeron en el estudio explicativo, prospectivo, transversal de casos y controles mujeres que asistieron a la consulta de Medicina Interna, Endocrinología y Ginecología del Hospital Central “Dr. Urquinaona” con diagnóstico de SOPQ. El Comité de Ética del hospital aprobó el estudio, y se obtuvo consentimiento por escrito de todas las mujeres.

El diagnóstico de SOPQ se confirmó por los siguientes criterios: evidencia de oligoanovulación (menos de 6 periodos menstruales en el año previo), signos clínicos o bioquímicos de hiperandrogenismo (concentraciones de testosterona plasmática por encima del límite superior normal y relación LH [lutoprina]/FSH [folitropina] anormal $>2$ ), y ovarios normales o aumentados de tamaño (>10 mL) con la presencia de microquistes subcapsulares (en número de 12 o más) de 2-9 milímetros de diámetro en la evaluación ecográfica abdominal (Trikudanathan, 2015).

Se seleccionaron mujeres con SOPQ y obesidad (índice de masa corporal > $30 \mathrm{Kg} / \mathrm{m}^{2} ;$ grupo $\mathrm{A}, \mathrm{n}=34$ ) y no obesas (índice de masa corporal $<25 \mathrm{~kg} / \mathrm{m}^{2}$; grupo $B, \mathrm{n}=13$ ). Las pruebas hormonales y la ecografía abdominal se realizaron durante la fase folicular temprana, entre el tercer y quinto día del ciclo menstrual espontáneo. El grupo control (grupo $C, n=47$ ) consistió en mujeres de edades similares con menstruaciones regulares y ovarios normales por ecografía, que asistieron a la consulta por patologías diferentes a SOPQ. Todos los controles se estudiaron del día 3 al 5 de su ciclo menstrual. Se excluyeron las mujeres con enfermedad tiroidea o suprarrenal, presencia de hiperprolactinemia, mujeres con hipertensión secundaria, insuficiencia renal con aclaramiento de creatinina $<30$ $\mathrm{mL} / \mathrm{min}$ por 1,73 $\mathrm{m}^{2}$ de superficie corporal, excreción de proteína urinaria mayor de $1 \mathrm{~g} /$ día, ángor pectoris, infarto del miocardio o enfermedad cerebrovascular reciente y a aquellas mujeres que no aceptaron participar en el estudio. Las mujeres que tomaban fármacos antihipertensivos fueron excluidas del estudio, y a las que tomaban fármacos hipolipemiantes se les solicitó que los suspendieran por 4 semanas antes del estudio. Ninguna paciente tomaba fármacos que afectaran las concentraciones de ADMA (por ejemplo, anticonceptivos orales o metformina).

El índice de masa corporal (IMC) se calculó por el peso dividido por la talla al cuadrado $\left(\mathrm{kg} / \mathrm{m}^{2}\right)$, mientras que la relación cintura cadera (RCC) se calculó por la división de la circunferencia de la cintura entre la circunferencia de la cadera. Se midió la circunferencia de la cintura y la cadera en la región más estrecha del abdomen y en la parte más ancha de la región glútea, respectivamente.

Todas las muestras de sangre venosa se tomaron en ayunas, en la primera semana posterior a la menstruación espontánea o inducida. Todas se manejaron de forma similar y se almacenaron a $-80^{\circ} \mathrm{C}$ por 1 a 3 días. Las concentraciones de FSH, LH, estradiol, androstendiona y testosterona se midieron por radioinmunoanálisis y quimioluminiscencia usando kits comerciales (Immulite 2000, Diagnostic Product Corp, EE.UU.). Los coeficientes de variación intra e inter ensayo fueron de 4 y $7 \%$ para $\mathrm{FSH}, 6$ y $7 \%$ para $\mathrm{LH}, 7$ y $9 \%$ para estradiol, 6 y $10 \%$ para androstenodiona y 4 y $7 \%$ para testosterona, respectivamente. La globulina fijadora de hormonas sexuales (GFHS) se cuantificó por inmunoensayo (Perkin-Elmer Auto-DELFIA Immunoassay analyzer); el coeficiente de variación inter ensayo fue de $3 \%$ e intra ensayo de $4 \%$, respectivamente. La glucosa sérica se cuantificó por el método de la glucosa-oxidasa 
Mejía et al.

(Pointe Scientific Inc., EE. UU.). Los coeficientes de variación intra e inter ensayo fueron 1,4 y 1,9\%. La insulina se determinó por radioinmunoanálisis (Coat-a-Count, Diagnostic Products Corp, EE.UU.). Los coeficientes de variación intra e inter ensayo fueron 1,6 y 5,5\%, respectivamente. Las concentraciones plasmáticas de ADMA fueron medidas por un método inmunoenzimático (DLD Diagnostika GMBH, Alemania). Los coeficientes de variación intra e inter ensayo de $6,0 \%$ y $0,4 \%$, respectivamente.

Los datos se presentan como media +/- desviación estándar. El análisis estadístico se realizó con la prueba de ANOVA con post prueba de Dunnett entre los grupos de mujeres con SOPQ (grupo $A$ y $B$ ), tomando como controles a las mujeres del grupo C. Los coeficientes de correlación entre las concentraciones del ADMA con los parámetros de laboratorio se evaluaron usando la prueba de Pearson. Se realizó un análisis de regresión lineal entre los diferentes parámetros de laboratorio y las concentraciones ADMA. Se consideró un valor $\mathrm{p}<0,05$ como estadísticamente significativo.

\section{Resultados}

Las características clínicas y endocrinas de las mujeres con SOPQ y los controles se muestran en la tabla 1. Los grupos eran similares en edad (23,2 +/- 2,8 años para el grupo $A$ y $24,0+/-3,7$ años para el grupo $B ; p=0,2403)$ e IMC $\left(30,6+/-5,1 \mathrm{Kg} / \mathrm{m}^{2}\right.$ para el grupo $A$ y $29,8+/-5,0$ para el grupo $B ; p=0,4445)$. Las concentraciones de $L H$, $\mathrm{FSH}$ y la relación $\mathrm{FSH} / \mathrm{LH}$ estaban significativamente más elevadas en las mujeres con SOPQ comparado con las mujeres del grupo control $(p<0,0001)$. No se encontraron diferencias estadísticamente significativas en las concentraciones de estradiol (52,3 +/- 5,1 pg/ $\mathrm{mL}$ para el grupo A y 53,2 +/-7,9 pg/mL; $p=0,5134)$. Los valores de testosterona y androstendiona fueron significativamente más altos en las mujeres con diagnóstico de SOPQ $(p<0,0001)$. Los valores de globulina fijadora de hormonas sexuales fueron significativamente menores en las mujeres con SOPQ comparado con los controles (1,6 +/- 0,3 ng/mL para el grupo A y 3,3 +/-0,4 ng/mL para el grupo $B ; p<0,0001)$. También se encontraron concentraciones más altas de insulina y glicemia en ayunas en las pacientes con SOPQ comparado con los controles ( $p<0,0001)$.

Los valores de ADMA se muestran en la tabla 1. Se observó que las mujeres con SOPQ mostraron concentraciones significativamente más altas (0,55 +/- 0,05 picomol/L) comparado con los valores promedios en las mujeres del grupo control $(0,46+/-0,03$ picomol/L; $\mathrm{p}<0,0001$ ).
Tabla 1: Características de las pacientes con síndrome de ovarios poliquísticos y controles.

\begin{tabular}{|c|c|c|c|}
\hline & $\begin{array}{l}\text { Paciente } \\
\text { con SOPQ } \\
(n=47)\end{array}$ & $\begin{array}{l}\text { Controles } \\
(n=47)\end{array}$ & $P$ \\
\hline Edad, años & $23,2+/-2,8$ & $24,0+/-3,7$ & 0,2403 \\
\hline $\begin{array}{l}\text { Índice de masa } \\
\text { corporal, } \mathrm{Kg} / \mathrm{m}^{2}\end{array}$ & $30,6+/-5,1$ & $29,8+/-5,0$ & 0,4445 \\
\hline Relación cintura cadera & $0,9+/-0,1$ & $0,9+/-0,1$ & 0,9999 \\
\hline Lutotropina, $\mathrm{mUl} / \mathrm{mL}$ & $9,6+/-3,1$ & $3,1+/-0,8$ & $<0,0001$ \\
\hline Folitropina, $\mathrm{mUI} / \mathrm{mL}$ & $6,3+/-0,9$ & $3,8+/-1,1$ & $<0,0001$ \\
\hline Relación FSH/LH & $0,8+/-0,4$ & $1,3+/-0,4$ & $<0,0001$ \\
\hline Estradiol, pg/ml & $52,3+/-5,1$ & $53,2+/-7,9$ & 0,5134 \\
\hline Testosterona, ng/ml & $5,0+/-1,2$ & $3,0+/-0,8$ & $<0,0001$ \\
\hline Andostendiona, ng/ml & $2,6+/-0,4$ & $1,9+/-0,5$ & $<0,0001$ \\
\hline $\begin{array}{l}\text { Globulina fijadora de } \\
\text { hormonas sexuales, } \mathrm{ng} / \mathrm{ml}\end{array}$ & $1,6+/-0,3$ & $3,3+/-0,4$ & $<0,0001$ \\
\hline $\begin{array}{l}\text { Insulina sérica en ayunas, } \\
\mathrm{mU} / \mathrm{L}\end{array}$ & $22,7+/-8,2$ & $6,2+/-0,6$ & $<0,0001$ \\
\hline $\begin{array}{l}\text { Glucosa sérica } \\
\text { en ayunas, mg/dl }\end{array}$ & $112,0+/-16,8$ & $94,4+/-9,0$ & $<0,0001$ \\
\hline $\begin{array}{l}\text { Dimetilarginina } \\
\text { asimétrica, picomol/L }\end{array}$ & $0,55+/-0,05$ & $3,4+/-1,0$ & $<0.0001$ \\
\hline
\end{tabular}

En la tabla 2 se observan las características de las mujeres con síndrome de ovarios poliquísticos obesas (grupo $A ; n=34$ ), mujeres con SOPQ y no obesas (grupo B; $n=13$ ), controles obesas (grupo $C ; n=33$ ) y controles no obesas (grupo $D ; n=14$ ). Las mujeres de los cuatro grupos no mostraron diferencias estadísticamente significativas con relación a la edad ( $p=n s)$. Las mujeres de ambos grupos de SOPQ (tabla 2) presentaron valores más elevados de LH, $\mathrm{FSH}$, relación $\mathrm{FSH} / \mathrm{LH}$, testosterona y androstendiona comparado con las mujeres de los grupos Cy D ( $<<0,0001)$. No se encontraron diferencias significativas en las concentraciones de estradiol entre las mujeres del grupo A y B comparado con las mujeres del grupo C y $D(p=0,6006$ y $p=0,7232$, respectivamente). Por otro lado, las concentraciones de globulina fijadora de hormonas sexuales fueron más bajas en ambos grupos de mujeres con diagnóstico de SOPQ comparado con los controles ( $p<0,0001$ ). Con respecto a las concentraciones de insulina, las mujeres de los grupos A y B presentaron concentraciones significativamente más altas que las mujeres del grupo C y D. Las mujeres con SOPQ obesas y no obesas presentaron concentraciones de glucosa sérica significativamente más altas que los controles obesos y no obesos, respectivamente $(p<0,0001)$. 
Se encontró que las mujeres con SOPQ y obesas tenían concentraciones de ADMA significativamente más altas que las controles obesos (0,56+/- 0,05 picomol/L comparado con 0,47 +/- 0,02 picomol/L; $\mathrm{p}<0,0001)$. De igual forma, las pacientes con SOPQ no obesas se observaron concentraciones significativamente más altas de ADMA al compararlas con las mujeres controles no obesas $(0,51+/$ - 0,03 picomol/L comparado con 0,45+/- 0,04 picomol/L; $\mathrm{p}<0,0001)$.
Al analizar el grupo de mujeres con SOPQ obesas y no obesas, se observó que las concentraciones del ADMA presentaba una correlación significativa con los valores de glicemia en ayunas $(r=0,114 ; p<0,0001)$ e insulina en ayunas $(r=0,062 ; p<0,0001)$. El análisis de regresión lineal mostró que el factor que afectaba la concentración plasmática del ADMA fueron las concentraciones de testosterona (beta $=0,013 ; p<0,05$ ), insulina en ayunas (beta $=0,002 ; p<0,05)$ y glucosa en ayunas (beta $=0,001 ; p<0,05$ ).

Tabla 2: Características de las pacientes con síndrome de ovarios poliquísticos y controles obesas y no obesas.

\begin{tabular}{|c|c|c|c|c|c|c|}
\hline & $\begin{array}{c}\text { GRUPO A } \\
\text { SOPQ } \\
\text { obesas } \\
(n=34)\end{array}$ & $\begin{array}{c}\text { GRUPO C } \\
\text { Controles } \\
\text { obesas } \\
(n=33)\end{array}$ & $p$ & $\begin{array}{c}\text { GRUPO B } \\
\text { SOPQ } \\
\text { no obesas } \\
(n=13)\end{array}$ & $\begin{array}{l}\text { GRUPO D } \\
\text { Controles } \\
\text { no obesas } \\
(n=14)\end{array}$ & $p$ \\
\hline Edad, años & $23,1+/-2,9$ & $24,0+/-3,6$ & 0,2632 & $23,6+/-2,8$ & $23,7+/-4,0$ & 0,9411 \\
\hline $\begin{array}{l}\text { Índice de masa } \\
\text { corporal, } \mathrm{Kg} / \mathrm{m}^{2}\end{array}$ & $33,5+/-1,8$ & $32,7+/-2,4$ & 0,1268 & $23,1+/-2,0$ & $23,0+/-1,2$ & 0,8750 \\
\hline Relación cintura cadera & $0,9+/-0,1$ & $0,9+/-0,1$ & 0,5243 & $0,8+/-0,1$ & $0,8+/-0,1$ & 0,5583 \\
\hline Lutotropina, mUI/mL & $9,2+/-3,1$ & $3,0+/-0,7$ & $<0,0001$ & $10,5+/-3,2$ & $3,0+/-0,8$ & $<0,0001$ \\
\hline Folitropina, mUI/mL & $6,3+/-0,9$ & $3,8+/-1,1$ & $<0,0001$ & $6,5+/-0,8$ & $3,9+/-0,9$ & $<0,0001$ \\
\hline Relación FSH/LH & $0,8+/-0,4$ & $1,3+/-0,5$ & $<0,0001$ & $0,7+/-0,4$ & $1,4+/-0,4$ & $<0,0001$ \\
\hline Estradiol, pg/ml & $52,4+/-5,3$ & $53,3+/-8,4$ & 0,6006 & $52,0+/-4,3$ & $52,8+/-6,9$ & 0,7232 \\
\hline Testosterona, ng/ml & $5,2+/-1,1$ & $2,9+/-0,8$ & $<0,0001$ & $4,4+/-1,2$ & $3,2+/-0,7$ & 0,0037 \\
\hline Andostendiona, ng/ml & $2,6+/-0,4$ & $1,9+/-0,5$ & $<0,0001$ & $2,5+/-0,3$ & $1,9+/-0,6$ & 0,0033 \\
\hline $\begin{array}{l}\text { Globulina fijadora de } \\
\text { hormonas sexuales, ng/ml }\end{array}$ & $1,6+/-0,4$ & $3,2+/-0,4$ & $<0,0001$ & $1,8+/-0,3$ & $3,6+/-0,4$ & $<0,0001$ \\
\hline $\begin{array}{l}\text { Insulina sérica en ayunas, } \\
\mathrm{mU} / \mathrm{L}\end{array}$ & $27,1+/-4,9$ & $6,1+/-0,5$ & $<0,0001$ & $11,4+/-1,7$ & $6,3+/-0,6$ & $<0,0001$ \\
\hline $\begin{array}{l}\text { Glucosa sérica } \\
\text { en ayunas, mg/dl }\end{array}$ & $115,7+/-13,1$ & $94,7+/-12,1$ & $<0,0001$ & $102,3+/-13,1$ & $93,7+/-9,9$ & 0,0251 \\
\hline $\begin{array}{l}\text { Dimetilarginina } \\
\text { asimétrica, picomol/L }\end{array}$ & $0,56+/-0,05$ & $0,51+/-0,03$ & $<0,0001$ & $0,47+/-0,02$ & $0,45+/-0,04$ & $<0,0001$ \\
\hline
\end{tabular}

\section{Discusión}

Los resultados de la investigación demuestran que las mujeres obesas y no obesas con SOPQ presentan concentraciones más elevadas de ADMA comparado con las mujeres controles. Estos resultados podrían demostrar que las mujeres con SOPQ tienen un riesgo elevado de disfunción endotelial.

La ADMA no procede directamente de la metilación de la arginina. Las dimetilargininas son moléculas que se forman como resultado de la proteólisis de proteínas con residuos de L-arginina metiladas. Al igual que otras metilargininas, se genera por modificaciones postranslacionales de residuos de arginina dentro de una variedad de proteínas específicas que predominantemente se encuentran en núcleos celulares (Alpoim et al., 2015). La metilación de los residuos de arginina es catalizada por un grupo de enzimas denominadas
S-adenosilmetionina N-metiltransferasas, proteínas metilasas I y II ya que transfieren uno o más grupos metilos desde el donador S-adenosilmetionina, producto intermediario del metabolismo de la homocisteína, a proteínas o polipéptidos con residuos de L-arginina (Hyun et al., 2000). Esta reacción produce $\mathrm{N}$-adenosil-L-homocisteína y proteínas metiladas que contienen residuos de L-arginina (proteínas con residuos de ADMA). La hidrólisis de las proteínas metiladas libera ADMA, que posteriormente pasa al plasma sanguíneo.

Estudios clínicos y epidemiológicos han demostrado que las altas concentraciones de ADMA están asociadas con enfermedades cardiovasculares (Kayacelebi et al., 2015). Sin embargo, existen pocos estudios sobre las concentraciones de ADMA en mujeres con SOPQ con resultados contradictorios. Heutling et al. (2008) demostraron que las mujeres con SOPQ tenían concentraciones elevadas de 
ADMA sugiriendo un aumento en el riesgo de enfermedades cardiovasculares, al demostrar que tenían concentraciones más elevadas de ADMA que el grupo control y esta diferencia continua siendo significativa luego de ajustar el IMC. Estas observaciones fueron confirmadas posteriormente por dos investigaciones (Kilic et al., 2011; Karakurt et al., 2014) reportaron concentraciones más altas en las mujeres con SOPQ que en los controles. Por otro lado, otros investigadores han reportado concentraciones similares de ADMA en adolescentes con SOPQ que en los controles (Demirel et al., 2007).

En la presente investigación, las concentraciones de insulina y glicemia en ayunas mostraron ser factores que modificaban las concentraciones de ADMA. En las mujeres con SOPQ, las concentraciones plasmáticas de ADMA se correlacionaron en forma positiva y significativa con el IMC, la insulina en ayunas y el péptido $C$ en ayunas (Heutling et al., 2008). Los hallazgos de la investigación confirman la asociación entre las concentraciones de ADMA con el índice de masa corporal y las concentraciones de glicemia e insulina en ayunas. Todos estos hallazgos apoyan la hipótesis que el ADMA puede representar un enlace entre la acción alterada de la insulina y la disfunción endotelial en el SOPQ, ya que la resistencia a la insulina representa un papel crucial en la patogénesis del SOPQ. Diferentes investigaciones han sugerido que tanto las mujeres obesas como no obesas con SOPQ tienen un aumento en la incidencia de resistencia a la insulina y de la diabetes no insulinodependiente. Además, el SOPQ y la obesidad tienen efectos negativos sinergísticos sobre la tolerancia a la glucosa (Celik et al., 2014).

Estudios previos han reportado concentraciones similares de ADMA en mujeres con SOPQ tanto obesas como no obesas, a pesar de las diferencias en insuilonoresistencia (Kilic et al., 2011; Turkcuoglu et al., 2011). Esto podría indicar que la contribución de diferentes factores, a parte de la obesidad, eleva el riesgo cardiovascular en estas mujeres. Los hallazgos de esta investigación son avalados por investigaciones previas que confirman la relación entre las concentraciones de ADMA y las concentraciones de glicemia e insulina (Nakhjavani et al., 2010). El mecanismo por el cual las concentraciones de glucosa e insulina modifican las concentraciones de ADMA es desconocido. Las fluctuaciones de los valores de glicemia e insulina pueden afectar la producción o el metabolismo del ADMA al afectar la actividad de la enzima dimetilarginina - dimetilaminohidrolasa en diferentes tejidos como el hígado y los riñónes (Trocha et al., 2014). Se han demostrado correlaciones significativas en las concentraciones de ADMA e insulina en pacientes jóvenes con diabetes mellitus tipo 1 (Marcovecchio et al., 2011).

Estudios previos han demostrado una relación entre las concentraciones de ADMA y la resistencia a la insulina (Alpoim et al., 2015). Sin embargo, no existe suficiente evidencia para indicar una asociación entre las concentraciones de ADMA y la aterosclerosis. Sen et al. (2009) demostraron que la acumulación de este inhibidor endógeno de la sintetasa de ON es un factor de riesgo importante de enfermedad cardiovascular en pacientes con insuficiencia renal crónica y sugiere la relación potencial entre el ADMA y la inflamación.

Morán et al. (2008) han demostrado que el hiperandrogenismo se correlaciona positivamente con la hiperinsulinemia. Estos hallazgos reflejan el posible efecto estimulador de las altas concentraciones de insulina sobre la biosíntesis tecal de testosterona y también prueba la contribución del hiperandrogenismo a la hiperinsulinemia (Galazis et al., 2011).

En el análisis de regresión, las concentraciones de testosterona parecen ser un determinante negativo en las concentraciones de ADMA. Existe escasa información o evidencia indirecta de la posible asociación entre las concentraciones de ADMA y las de testosterona, ya que la disfunción endotelial ha sido asociada con un incremento de las concentraciones de andrógenos en mujeres obesas con SOPQ (Moran et al. 2009). Sin embargo, se ha demostrado que las concentraciones ADMA pueden ser modificadas por las hormonas sexuales y se ha encontrado que la testosterona incrementa las concentraciones de ADMA en transexuales femeninos a masculinos (Bunck et al., 2009). Por otra parte, también se ha demostrado que la función endotelial sufre mediciones durante el ciclo menstrual (Adkisson et al. 2010), por lo que no se pudo realizar una recolección estandarizada de las muestras de sangre en momentos específicos del ciclo debido a la variabilidad de la duración de los ciclos en las mujeres con SOPQ.

Se ha sugerido que la mayoría de los factores de riesgo cardiovascular ejercen sus efectos negativos sobre la función endotelial a través de una vía patogénica común que lleva a una acumulación del ADMA, un potente inhibidor de la sintasa de ON, lo cual provoca alteraciones de este al disminuir la vasodilatación y favorecer la agregación plaquetaria, la proliferación celular, la oxidación de las LDL, la aparición de radicales libres y otros factores que contribuyen a la formación y progresión del proceso aterosclerótico (Tousoulis et al., 2015). Más aún, las concentraciones de ADMA han sido propuestas como un factor de riesgo cardiovascular independiente (Bouras et al., 2016). Por lo tanto, es posible que la disfunción endotelial asociada al SOPQ, reflejada por las altas concentraciones de ADMA, podría ser sea responsable del aumento de la morbilidad cardiovascular.

Estas observaciones aportan evidencia que permite concluir que existe aumento en las concentraciones plasmáticas de dimetilarginina asimétrica en las mujeres con SOPQ obesas y no obesas comparado 
Mejía et al.

con las mujeres controles sanas. Además, las alteraciones de las concentraciones séricas se relacionan con las modificaciones en las concentraciones de glicemia e insulina en ayunas.

\section{Declaración de aspectos éticos}

Reconocimiento de autoría: Todos los autores declaran que han realizado aportes a la idea, diseño del estudio, recolección de datos, análisis e interpretación de datos, revisión crítica del contenido intelectual y aprobación final del manuscrito que estamos enviando. Responsabilidades éticas: Protección de personas. Los autores declaran que los procedimientos seguidos se conformaron a las normas éticas del comité de experimentación humana responsable y de acuerdo con la Asociación Médica Mundial y la Declaración de Helsinki. Confidencialidad de los datos: Los autores declaran que han seguido los protocolos del Hospital Central “Dr. Urquinaona" sobre la publicación de datos de pacientes. Derecho

a la privacidad y consentimiento informado: Los autores han obtenido el consentimiento informado de los pacientes $y / o$ sujetos referidos en el artículo. Este documento obra en poder del autor de correspondencia. Financiamiento: Los autores certifican que no han recibido apoyos financieros, equipos, en personal de trabajo o en especie de personas, instituciones públicas y/o privadas para la realización del estudio. Conflicto de intereses: Los autores declaran no tener ningún conflicto de intereses.

\section{Referencias}

Adkisson EJ, Casey DP, Beck DT, Gurovich AN, Martin JS \& Braith RW. (2010). Central, peripheral and resistance arterial reactivity: fluctuates during the phases of the menstrual cycle. Experimental Biology and Medicine (Maywood) 235, 111-118.

Alpoim PN, Sousa LP, Mota AP, Rios DR \& Dusse LM. (2015). Asymmetric Dimethylarginine (ADMA) in cardiovascular and renal disease. Clinica Chimica Acta 440, 36-39.

Ari H, Ari S, Erdoğan E, Tiryakioğlu O, Ustündağ Y, Huysal K, Koca V \& Bozat T. (2010). A novel predictor of restenosis and adverse cardiac events: asymmetric dimethylarginine. Heart and Vessels 25, 19-26.

Arikan S, Akay H, Bahceci M, Tuzcu A \& Gokalp D. (2009). The evaluation of endothelial function with flow-mediated dilatation and carotid intima media thickness in young nonobese polycystic ovary syndrome patients; existence of insulin resistance alone may not represent an adequate condition for deterioration of endothelial function. Fertility and Sterility 91, 450-455.
Atzeni F, Sarzi-Puttini P, Sitia S, Tomasoni L, Gianturco L, Battellino M, Boccassini L, De Gennaro Colonna V, Marchesoni A \& Turiel M. (2011). Coronary flow reserve and asymmetric dimethylarginine levels: new measurements for identifying subclinical atherosclerosis in patients with psoriatic arthritis. The Journal of Rheumatology 38, 1661-1664.

Bouras G, Deftereos S, Tousoulis D, Giannopoulos G, Chatzis G, Tsounis D, Cleman MW \& Stefanadis C. (2013). Asymmetric Dimethylarginine (ADMA): a promising biomarker for cardiovascular disease? Current Topics in Medicinal Chemistry 13, 180-200.

Bunck M, Giltay E, Diamant M, Gooren L \& Teerlink T. (2009). Differential effects of cross-sex hormonal treatment on plasma asymmetric dimethylarginine (ADMA) in healthy male-to-female and female-to-male transsexuals. Atherosclerosis 206; 245-250..

Celik C, Tasdemir N, Abali R, Bastu E \& Yilmaz M. (2014). Progression to impaired glucose tolerance or type 2 diabetes mellitus in polycystic ovary syndrome: a controlled follow-up study. Fertility and Sterility 101, 1123-1128.

Cobin RH. (2013). Cardiovascular and metabolic risks associated with PCOS. Internal and Emergency Medicine 8 Suppl 1, S61-S64. Demirel F, Bideci A, Cinaz P, Camurdan MO, Biberoğlu G, Yesilkaya E \& Hasanoğlu A. (2007). Serum leptin, oxidized low density lipoprotein and plasma asymmetric dimethylarginine levels and their relationship with dyslipidaemia in adolescent girls with polycystic ovary syndrome. Clinical Endocrinology 67, 129-134.

Dimitroulas T, Sandoo A \& Kitas GD. (2012). Asymmetric dimethylarginine as a surrogate marker of endothelial dysfunction and cardiovascular risk in patients with systemic rheumatic diseases. International Journal of Molecular Sciences 13, 12315-12335.

Galazis N, Galazi M \& Atiomo W. (2011). D-Chiro-inositol and its significance in polycystic ovary syndrome: a systematic review. Gynecological Endocrinology 27, 256-262.

Heutling D, Schulz H, Nickel I, Kleinstein J, Kaltwasser P, Westphal S, Mittermayer F, Wolzt M, Krzyzanowska K, Randeva H, Schernthaner G \& Lehnert H. (2008). Asymmetrical dimethylarginine, inflammatory and metabolic parameters in women with polycystic ovary syndrome before and after metformin treatment. The Journal of Clinical Endocrinology \& Metabolism 93, 82-90.

Hyun YL, Lew DB, Park SH, Kim CW, Paik WK \& Kim S. (2000). Enzymic methylation of arginyl residues in -gly-arg-gly-peptides. Biochemical Journal 348, 573-578. 
Mejía et al.

Karakurt F, Carlioglu A, Kaygusuz I, Gumus II, Uz B \& Akdeniz D. (2014). Effect of ethinyl estradiol-cyproterone acetate treatment on asymmetric dimethyl-arginine levels in women with polycystic ovary syndrome. Archives of Gynecology and Obstetrics 289, 135-140.

Kayacelebi AA, Willers J, Pham VV, Hahn A, Schneider JY, Rothmann S, Frölich JC \& Tsikas D. (2015). Plasma homoarginine, arginine, asymmetric dimethylarginine and total homocysteine interrelationships in rheumatoid arthritis, coronary artery disease and peripheral artery occlusion disease. Amino Acids 47, 1885-1891.

Kilic S, Yilmaz N, Zulfikaroglu E, Erdogan G, Aydin M \& Batioglu S. (2011). Inflammatory-metabolic parameters in obese and nonobese normoandrogenemic polycystic ovary syndrome during metformin and oral contraceptive treatment. Gynecological Endocrinology 27, 622-629.

Lind L, Ingelsson E, Kumar J, Syvänen AC, Axelsson T \& Teerlink T. (2013). Genetic variation in the dimethylarginine dimethylaminohydrolase 1 gene (DDAH1) is related to asymmetric dimethylarginine (ADMA) levels, but not to endothelium-dependent vasodilation. Vascular Medicine 18, 192-199.

Marcovecchio M, Widmer B, Turner C, Dunger D \& Dalton R. (2011). Asymmetric dimethylarginine in young people with Type 1 diabetes: a paradoxical association with $\mathrm{HbA}(1 \mathrm{c})$. Diabetic Medicine 28, 685-691.

Moran LJ, Hutchison SK, Meyer C, Zoungas S \& Teede HJ. (2009). A comprehensive assessment of endothelial function in overweight women with and without polycystic ovary syndrome. Clinical Science 116, 761-770.

Moran C, Renteria JL, Moran S, Herrera J, Gonzalez S \& Bermudez JA. (2008). Obesity differentially affects serum levels of androstenedione and testosterone in polycystic ovary syndrome. Fertility and Sterility 90, 2310-2317.

Nakhjavani M, Karimi-Jafari H, Esteghamati A, Khalilzadeh O, Asgarani F \& Ghadiri-Anari A. (2010). ADMA is a correlate of insulin resistance in early-stage diabetes independent of hs-CRP and body adiposity. Annales d'Endocrinologie 71, 303-308..
Sen N, Poyraz F, Tavil Y, Yazici HU, Turfan M, Hizal F, Topal S, Erdamar $\mathrm{H}$, Cakir E, Yalçin R \& Cengel A. (2009). Carotid intima-media thickness in patients with cardiac syndrome $X$ and its association with high circulating levels of asymmetric dimethylarginine. Atherosclerosis 204, e82-e85.

Shah D \& Rasool S. (2016).Polycystic ovary syndrome and metabolic syndrome: the worrisome twosome? Climacteric 19, 7-16.

Tousoulis D, Georgakis MK, Oikonomou E, Papageorgiou N, Zaromitidou M, Latsios G, Papaioannou S \& Siasos G. (2015). Asymmetric Dimethylarginine: Clinical Significance and Novel Therapeutic Approaches. Current Medicinal Chemistry 22, 2871-2901.

Trikudanathan S. (2015). Polycystic ovarian syndrome. Medical Clinics of North America 99, 221-235.

Trocha M, Merwid-Ląd A, Chlebda-Sieragowska E, Szuba A, Pieśniewska M, Fereniec-Gołębiewska L, Kwiatkowska J, Szeląg A \& Sozański T. (2014). Age-related changes in ADMA-DDAH-NO pathway in rat liver subjected to partial ischemia followed by global reperfusion. Experimental Gerontology 50, 45-51.

Türkçüoğlu I, Engin-Üstün Y, Turan F, Kali Z, Karabulut AB, Meydanli M, Kafkasli A (2011). Evaluation of asymmetric dimethylarginine, nitric oxide levels and associated independent variables in obese and lean patients with polycystic ovarian syndrome. Gynecological Endocrinology 27, 609-614.

Vipin VP, Dabadghao P, Shukla M, Kapoor A, Raghuvanshi AS \& Ramesh V. (2016). Cardiovascular disease risk in first-degree relatives of women with polycystic ovary syndrome. Fertility and Sterility $105,1338-1344$.

Willeit P, Freitag DF, Laukkanen JA, Chowdhury S, Gobin R, Mayr M, Di Angelantonio E \& Chowdhury R. (2015). Asymmetric dimethylarginine and cardiovascular risk: systematic review and meta-analysis of 22 prospective studies. Journal of the American Heart Association 4, e001833.

Zhang HY, Guo CX, Zhu FF, Qu PP, Lin WJ \& Xiong J. (2013). Clinical characteristics, metabolic features, and phenotype of Chinese women with polycystic ovary syndrome: a large-scale case-control study. Archives of Gynecology and Obstetrics 287, 525-531. 